\title{
Towards salutogenesis in the development of personalised and preventive healthcare
}

\author{
Mauro Alivia • Paola Guadagni • \\ Paolo Roberti di Sarsina
}

Received: 1 August 2011 / Accepted: 13 October 2011 /Published online: 8 November 2011

(C) European Association for Predictive, Preventive and Personalised Medicine 2011

\begin{abstract}
The purpose of this review is to discuss how a salutogenetic approach that takes into consideration the human being as physical, psychological and spiritual entity may provide some answers to the difficulties faced by healthcare systems. The choice of medical intervention needs to take into account the technological advances of biomedicine but tailor them to the physical, psychological and spiritual needs of the patient in the context of their biography. Such person-centred medicine aims to strengthen Antonovsky's concepts of resilience and sense of coherence with each therapeutic intervention so that overcoming illness becomes the foundation for better future health. Appropriate evaluation parameters need to be developed and included in order to evaluate the success of interventions in a personcentred, salutogenetic system.
\end{abstract}

Keywords Personalised medicine - Preventive medicine . Person-centred medicine $\cdot$ Salutogenesis $\cdot$ Non-conventional medicine

\author{
M. Alivia \\ Past President Italian Society of Antroposophic Medicine (SIMA), \\ Milan, Italy \\ M. Alivia $\cdot$ P. Guadagni $\cdot$ P. Roberti di Sarsina \\ Charity "Association for Person Centred Medicine", \\ Bologna, Italy \\ P. Roberti di Sarsina \\ Expert for non-conventional medicine, High Council of Health, \\ Ministry of Health, \\ Rome, Italy \\ M. Alivia $(\bowtie)$ \\ Corso di Porta Romana 118, \\ 20122 Milano, Italy \\ e-mail: mauroalivia@medicinaantroposofica.it
}

\section{Salutogenetic healthcare}

Illness is a challenge to our physical, psychological and spiritual wellbeing that has repercussions on our identity and our social context. In a pathogenetic approach, diagnostic tests are used to look for the underlying disease and treatments are aimed at removing it. A person is considered cured when the disease is no longer detectable and agreed parameters have normalised. However daily clinical practice is characterised by people who suffer from chronic illness where there is often a discrepancy between their biochemical results and the way they feel. Good or even normal parameters do not necessarily correlate with a good perceived state of health, and vice versa. Health is therefore a concept that goes beyond the absence of disease to include physical, mental and social wellbeing [1]. Salutogenesis, developed by Antonovsky in the 1970s, looks at what generates health by exploring the reasons why some people stay healthy in the face of hazardous influences whilst others, faced with the same hardship fall ill. Antonovsky's research shows how adverse events and stress can become the opportunity to generate health if certain personal characteristics are present. Resilience to difficult situations depends on a person's Sense of Coherence (SOC), a global orientation towards life that is based on self-reliance in the face of challenges, selfconfidence in one's ability to deal with demanding events and the trust that difficult events hold meaning for one's life $[2,3]$. There is a growing body of research on all age groups [4-6], different socioeconomic backgrounds and across cultures [7] that shows how a strong SOC is related to better health, healthier ageing [8-12] and is a protective factor against alcohol addiction despite similar rates of recreational consumption in teenagers [13]. Conversely, a weak SOC is related to poorer health and lower mood 
$[4,5]$. Although SOC develops naturally in the first 30 years of life it is not a static orientation. It can be strengthened through personal activity and care $[4,14]$.

A dynamic, personally driven element that can change through activity and care is thus added to the concept of health. It is not the absence of hardship or disease that determines health but our ability to deal with them positively and assign meaning to them. Conversely the presence of disease does not necessarily preclude wellbeing or fulfilment. Health is not a steady state, effortlessly maintained once it has been achieved, but an active process of continuous adjustment, a subtle equilibrium between our physiological, psychological and spiritual integrity and the outer or inner influences that can strengthen or undermine this. The concept of health as a state of "complete well-being" advocated by the Declaration of Alma Ata, becomes health as "the ability to adjust and self-manage" in the face of physical, emotional or personal challenges [15].

A salutogenetic healthcare system needs to be oriented in such a way that illness is considered an adverse event that can become the foundation for better future health. Methods need to be adopted that allow the ill person to be treated in all their characteristics and in context. Each medical intervention should aim to strengthen SOC and a person's physiological, psychological and spiritual resilience. The increasing prevalence of chronic diseases, the very different but constant environmental challenges faced by people in the developing and developed world alike, provide us with constant salutogenetic opportunities.

\section{Person-centred medicine and salutogenetic healthcare}

Person-centred medicine (PCM) takes into account the physical, psychological and spiritual aspects of a person in health and illness in order to individualise health promotion practices, diagnosis and treatment. It broadens the technological advances of Biomedicine with the epistemological approach, the relationship-based care and the salutogenetic treatments of non-conventional Medicine [16]. Complementary and alternative medicine (CAM) and traditional medicine (TM) are grouped together as $\mathrm{CAM} / \mathrm{TM}$ or non-conventional medicine (NCM). The term includes a variety of different medical systems and healthcare methods whose roots lie in different philosophical backgrounds and cultural origins. Although very different from one another, they all share a holistic view of the human being as a physical, psychological and spiritual entity. They value the complexity of natural phenomena and study the relationship between the human being and nature in health and illness. Their treatment systems are based on knowledge, skills and practices that restore health and encourage the self-healing abilities of the human being. They advocate respect for the dignity of every person and promote responsibility for keeping healthy at individual and community levels [17]. This holistic, personcentred, salutogenetic approach is the reason for the growing interest in all forms of NCM by patients and healthcare workers alike [18]. Salutogenetic healthcare practices are personalised using the principles and methods of personcentred medicine.

In salutogenetic healthcare the caregiver-person relationship needs to guide the therapeutic process at all times. Each professional role needs to be developed to provide salutogenetic care from as early as undergraduate training. In order to have a person-centred approach, technological proficiency, solid grounding in evidence-based medicine (EBM) and knowledge of international protocols need to be complemented by knowledge of the epistemological systems and practical tools of NCM [19]. Doctors and other healthcare workers are then encouraged to the use their clinical judgment as the final decision-making tool. Clinical judgment is central to the role of the doctor and their professionalism and can be strengthened and developed to become an accurate decision-making tool. This complements the application of EBM and protocols, which are based on large numbers and therefore not always applicable to particular cases. Following protocols ensures a basic standard of care that has lead to improvements in safety on a large scale. However following them automatically can lead to repetitive, monotonous practice which in turn can lead to errors as well as frustration and dissatisfaction both on the part of the healthcare worker, who feels like a generic $\operatorname{cog}$ in a smooth machine, and the patient who does not feel personally addressed. On the contrary, assessing and treating each person in their physical, psychological and spiritual uniqueness, encourages active personal involvement on the part of the both healthcare worker and the patient. The use of sound clinical judgment allows personalisation of guidelines and protocols to each case. It leads to more accurately placed resources and therefore less waste. Moreover, learning and teaching about salutogenetic practices may mean that healthcare workers adopt healthier lifestyles for themselves. Greater work satisfaction and active involvement strengthen a healthcare worker's resilience and SOC, ultimately improving their health as well as that of their patients. With this person-centred method, any treatment is chosen as the result of an informed choice that takes all aspects of the person into consideration.

For any therapeutic process to become a salutogenetic experience, physical as well as psychological, social and personal factors need to be addressed at the appropriate time with attentive care to the physical emotional and spiritual needs of the person. The patient needs to be 
able to rely on the doctor or the team who take responsibility for their treatment and recovery. During rehabilitation or at other times during the process there may be a chance to bring the experience to greater consciousness, to explore the reasons that led to the event and the things that could be changed in order to prevent similar episodes from happening again. New skills can be learned with activities such as cooking classes and artistic therapies. All this works towards developing an awareness that overcoming this difficult experience was meaningful because it allowed the development of new life skills, new coping strategies, and new confidence in one's own healing abilities [20]. Gradually the person takes on increasing amounts of responsibility for their own health. This change needs to be sustained over time with appropriate follow-up as habits are difficult to change and may slip if they are not followed closely and encouraged over time [21].

In order to evaluate the success of medical interventions broadened with a salutogenetic approach, parameters need to be developed and integrated into the current evaluation systems. They need to have a suitable epistemological basis, a methodological approach that considers the value of small numbers, individual cases and clinical judgement [22]. Salutogenetic parameters such as the quality of life of patients and caregivers, sustainable change, treatment satisfaction, job satisfaction and prevention of burn-out need to be assessed as well as cost-effectiveness and technological excellence [23].

\section{Personalised health promotion as disease prevention in salutogenetic healthcare}

Health education is both a therapeutic intervention and the key form of disease prevention in salutogenetic healthcare. It should take place at population levels, in primary care, in hospitals, as part of any rehabilitation process, in schools and families. Environmental obstacles that prevent people from adopting healthy lifestyles should be removed as far as possible. The Ottawa Charter advocates the implementation of eight conditions that should underlie any change in health. These are peace, shelter, education, food, income, a stable eco-system, sustainable resources, social justice, and equity. At the same time, individuals need to be encouraged to actively increase control over their health in order to improve it. "To reach a state of complete physical, mental and social well-being, an individual or group must be able to identify and to realize aspirations, to satisfy needs, and to change or cope with the environment" [24].

The quality of foodstuffs for health is the first topic to be addressed. Encouraging locally sourced, high quality foods, encouraging biodiversity and fair-trade, has positive effects on the sustainability of primary resources and the stability of the eco-system as well as people's health [25]. In order to be adopted by individuals in their lifestyles, these general considerations need to be adapted to individual situations. They need to be made personcentred. There should be variations between the diet of a child and that of an elderly person, of an office worker compared to that of a builder, the diet of someone who suffers from inflammatory illnesses compared to that of someone who suffers from cancer, the diet of the same person when they are well or ill. If diet were a better used resource in healthcare and people were taught its principles and practical applications, this may decrease the need to use medication in some cases.

The care of biorhythms is another aspect to be addressed. Altered sleeping patterns, eating rhythms and patterns of physical activity are both a measure and a cause of pathology and health. Variable shift workers have a higher degree of stress, of alcohol misuse and increased use of neuroleptic drugs compared to their colleagues with regular working patterns [26]. Many people have altered sleeping patterns because of habit, work or illness. Many others have altered eating rhythms as they skip meals, or constantly pick at food during the day. The benefits of regular exercise are well known to all. It is important for people to become aware of their own biorhythms, which alter with age, gender, state of health, personal and constitutional characteristics. With the help of education, improved environmental conditions and appropriate care during illness, people can adjust or at least compensate for altered biorhythms and therefore actively improve their health.

The emotional factors that contribute to health and illness also need to be addressed. Negative emotions increase the incidence of physical illness. Conversely positive emotions and humour improve health and wellbeing [11]. Chronic stress can strengthen or weaken a person depending on how strong or weak their resilience and SOC are. Chronic stress has been shown to be greater risk factor for developing ischaemic heart disease than hypertension or hypercholesterolaemia [20]. Emotions can have adverse effects on the incidence of complication and prognosis during an acute event. Apart from reducing known risk factors and encouraging positive emotions, we can strengthen emotional resilience and SOC through artistic activity in the form of painting, music, dance, drama, creative writing. A study carried out in Sweden showed how music therapy improved quality of life and SOC in a group of elderly people [10]. Through artistic activities people learn new skills and their achievements have a positive effect on their self-esteem. This can be applied to overcome future challenges or unknown lifesituations with strengthened SOC. 


\section{Outlook}

The initial steps towards a salutogenetic healthcare reform, are to broaden undergraduate training and to develop pilot projects in the fields of health promotion and salutogenetic rehabilitation. The person and the caregiver-person relationship needs to be placed at the centre of the therapeutic process through the principles of person-centred medicine both in education and in practice. Caregivers need to be given the tools to consider the person in all their aspects and characteristics through the adoption of the epistemological basis of NCM that broadens the technological advances of Biomedicine. Medical interventions should not only be aimed at removing the disease but also at improving health by strengthening a person's SOC and their resilience at physical, psychological and spiritual levels. Reasons for illness can be explored during rehabilitation. Through health promotion programmes, during rehabilitation, in education, people are taught how to take better care of themselves by adopting individually tailored, healthier lifestyles better suited to their own physiological, emotional and personal needs. This will allow them to maintain a steadier health equilibrium. If illness does occur, overcoming it can become an event that holds meaning. It can become the starting point for acquiring new strength and skills. SOC and resilience to deal with difficult situations are strengthened. Active involvement needs to be encouraged both on the part of the to patient and healthcare giver, the use of clinical judgment needs to be taught. Methods for the scientific investigation of effects on small numbers or single cases need to be included in the evaluation of a person-centred, salutogenetic healthcare system alongside EBM.

\section{References}

1. Declaration of Alma-Ata. International Conference on Primary Health Care. Alma-Ata, USSR; 1978.

2. Antonovsky A. Unravelling the mystery of health: how people manage stress and stay well. 1st ed. San Francisco: Jossey-Bass; 1987.

3. Johnson M. Approaching the salutogenesis of sense of coherence: the role of 'active' self-esteem and coping. Br J Health Psychol. 2004;9:419-32.

4. Feldt T, Kinnunen U, Mauno S. A mediational model of sense of coherence in the work context: a one-year follow-up study. J Organ Behav. 2000;21:461-76.

5. Anderson Darling C, McWey LM, Howard SN, Olmstead SB. College student stress: the influence of interpersonal relationships on sense of coherence. Stress Heal. 2007; doi:10.1002/smi.1139.

6. Fagerstrom L, Gustafson Y, Jakobsson G, Johansson S, Vartiainen P. Sense of security amongst people aged 65 and 75: external and inner sources of security. J Adv Nurs. 2011; doi:10.1111/j.13652648.2010.05562.x.

7. Bowman BJ. Cross-cultural validation of Antonovsky's sense of coherence scale. J Clin Psychol. 1996;52(5):547-9.

8. Diener E, Chan MY. Happy people live longer: subjective well-being contributes to health and longevity. Appl Psychol Health Wellbeing. 2011; doi:10.1111/j.1758-0854.2010.01045.x.

9. Söderhamn O, Holmgren L. Testing Antonovsky's sense of coherence (SOC) scale among Swedish physically active older people. Scand J Psychol. 2004;45:215-21.

10. Lee YY, Chan MF, Mok E. Effectiveness of music intervention on the quality of life of older people. J Adv Nurs. 2010; doi:10.1111/J.1365-2648.2010.05445.X.

11. Horowitz S. Effect of positive emotions on health-hope and humor. Alternative Compl Ther. 2009;15(4):196-202.

12. Lutgendorf S, Vitaliano P, Tripp-Reimer T, Harvey JH, Lubaroff DM. Sense of coherence moderates the relationship between life-stress and natural killer cell activity in healthy older adults. Psychol Aging. 1999;14:552-63.

13. Nilsson KW, Starrin B, Simonsson B, Leppert J. Alcohol- related problems among adolescents and the role of a sense of coherence. Int J Soc Welfare. 2007;16:159-67.

14. Bäärnhielm S. Restructuring illness meaning through the clinical encounter: a process of disruption and coherence. Cult Med Psychiatry. 2004;28:41-65.

15. Huber M, Knottnerus JA, Green L, Van der Horst H, Jadad AR, Kromhout D, et al. How should we define health? BMJ. 2011; doi:10.1136/bmj.d4163.

16. Roberti di Sarsina P. The social demand for a medicine focused on the person: the contribution of CAM to healthcare and healthgenesis. Evid Based Complement Alternat Med. 2007; doi:10.1093/ecam/nem094.

17. Rakel DP, Guerrera MP, Bayles BP, Desai GJ, Ferrara E. CAM education: promoting a salutogenic focus in health care. J Altern Complement Med. 2008; doi:10.1089/acm.2007.0562.

18. Roberti di Sarsina P, Iseppato I. Person-centred medicine: towards a definition. Forsch Komplementmed. 2010; doi:10.1159/ 000320603

19. Roberti di Sarsina P, Iseppato I. Traditional and non-conventional medicines: the socio-anthropological and bioethical paradigms for person-centred medicine, the Italian context. EPMA J. 2011; doi:10.1007/s13167-011-0104-z.

20. Giallauria F, Battimiello V, Veneziano M, De Luca P, Cipollaro I, Buonincontro $\mathrm{M}$, et al. Psychosocial risk factors in cardiac practice. Monaldi Arch Chest Dis. 2007;68:74-80.

21. Karlsson I, Berglin E, Larsson PA. Sense of coherence: quality of life before and after coronary artery bypass surgery: a longitudinal study. J Adv Nurs. 2000;31(6):1383-92.

22. Kienle GS, Kiene H. Clinical judgement and the medical profession. J Eval Clin Pract. 2011; doi:10.1111/j.1365-2753.2010.01560.x.

23. Ventegodt S, Omar HA, Merrick J. Quality of life as medicine: interventions that induce salutogenesis. A review of the literature. Soc Indic Res. 2011; doi:10.1007/s11205-010-9621-8.

24. The Ottawa charter for health promotion. Ottawa, Ontario, Canada: International Conference On Health Promotion; 1986.

25. Huber M, van de Vijver LPL, Parmentier H, Savelkoul H, Coulier $\mathrm{S}$, Wopereis $\mathrm{S}$, et al. Effects of organically and conventionally produced feed on biomarkers of health in a chicken model. Br J Nutr. 2010;103:663-76.

26. Gordon NP, Cleary PD, Parker CE, Czeisler CA. The prevalence and health impact of shiftwork. Am J Public Health. 1986;76 (10):1225-8. 